\title{
Reflets
}

Revue d'intervention sociale et communautaire

\section{Développement des connaissances sur les enjeux liés à la violence faite aux femmes en Ontario français - Deux cas de figure : la prostitution et l'accès aux services en droit de la famille}

\author{
Marie-Luce Garceau, Ghislaine Sirois et Marc Charron
}

Volume 21, numéro 1, printemps 2015

Violences faites aux femmes et contextes minoritaires

URI : https://id.erudit.org/iderudit/1032550ar

DOI : https://doi.org/10.7202/1032550ar

Aller au sommaire du numéro

Éditeur(s)

Reflets, Revue d'intervention sociale et communautaire

ISSN

1203-4576 (imprimé)

1712-8498 (numérique)

Découvrir la revue

Citer cet article

Garceau, M.-L., Sirois, G. \& Charron, M. (2015). Développement des connaissances sur les enjeux liés à la violence faite aux femmes en Ontario

français - Deux cas de figure : la prostitution et l'accès aux services en droit de la famille. Reflets, 21(1), 106-136. https://doi.org/10.7202/1032550ar
Résumé de l'article

Cet article porte sur le développement des connaissances dans le réseau de la violence faite aux femmes en Ontario français. S'ancrant dans une épistémologie résolument féministe, cette construction collective du savoir au sujet des enjeux auxquels sont confrontées les intervenantes en violence faite aux femmes s'élabore principalement au moyen de mécanismes de concertation. À ce jour, il existe peu d'articles sur la mise en place de tels mécanismes qui nourrissent les pratiques d'intervention et mènent à l'action. C'est pour combler cette lacune que nous proposons cette analyse exploratoire d'un sujet qui s'avère important quand on veut participer au développement souhaité et préparer la relève.
Tous droits réservés (C) Reflets, Revue d’intervention sociale et communautaire, 2015 ce document est protége par la loi sur le droit d'auteur. L'utilisation des services d’Érudit (y compris la reproduction) est assujettie à sa politique d'utilisation que vous pouvez consulter en ligne. 


\title{
Développement des connaissances sur les enjeux liés à la violence faite aux femmes en Ontario français - Deux cas de figure : la prostitution et l'accès aux services en droit de la famille
}

\author{
Marie-Luce Garceau \\ Professeure, École de service social, Université Laurentienne \\ Ghislaine Sirois \\ Chercheuse communautaire et activiste féministe, Gatineau \\ Marc Charron \\ Professeur, Université Laurentienne
}

\section{Résumé}

Cet article porte sur le développement des connaissances dans le réseau de la violence faite aux femmes en Ontario français. S'ancrant dans une épistémologie résolument féministe, cette construction collective du savoir au sujet des enjeux auxquels sont confrontées les intervenantes en violence faite aux femmes s'élabore principalement au moyen de mécanismes de concertation. À ce jour, il existe peu d'articles sur la mise en place de tels mécanismes qui nourrissent les pratiques d'intervention et mènent à l'action. C'est pour combler cette lacune que nous proposons cette analyse exploratoire d'un sujet qui s'avère 
important quand on veut participer au développement souhaité et préparer la relève.

Mots clés : femmes, francophones, Ontario français, services en français, violence faite aux femmes (VFF), prostitution, services juridiques, droit de la famille

\section{Abstract}

This article discusses the development of knowledge within organizations working in French in the field of violence against women in Ontario.Anchored in a resolutely feminist epistemology, this collective knowledge about issues identified by the frontline workers is built through various consultation mechanisms. To this day, very little research has been published concerning the development of such mechanisms aimed at enriching practice intervention. Thus the purpose of the following article, in which the authors propose a preliminary exploration of a subject of importance for service development and the preparation of the next generation of front-line workers.

Key words: women, Ontario francophones, violence against women, prostitution, judicial services, family law

\section{Introduction}

Le présent article porte sur le développement des connaissances dans le réseau de la violence faite aux femmes (VFF) en Ontario français. S'ancrant dans une épistémologie résolument féministe, cette construction collective du savoir au sujet des enjeux auxquels sont confrontées les intervenantes enVFF s'élabore principalement par le mécanisme de la concertation. À ce jour, peu d'articles ont été écrits sur la mise en place de tels mécanismes qui nourrissent les pratiques d'intervention et mènent à l'action. Notre analyse vise donc à combler cette lacune. Pour ce faire, nous nous pencherons particulièrement sur le mécanisme des rencontres bisannuelles 
des intervenantes comme lieu de concertation où se développent et s'échangent les connaissances autour des enjeux vécus et perçus. Puis, nous montrerons à partir de deux cas de figure, soit ceux de la prostitution et de l'accès à des services juridiques en français en droit de la famille, comment se tisse ce savoir propre à alimenter les pratiques et les interventions en matière de VFF en Ontario français.

Avant d'aborder le mécanisme du développement des connaissances autour des enjeux, précisons que par " enjeux " nous entendons à la fois les problèmes auxquels sont confrontées sur le terrain les intervenantes et les usagères dans le réseau d'organismes en VFF de l'Ontario français, de même que la recherche de solutions qui tiennent compte de l'intérêt des femmes aux prises avec ces derniers. Par ailleurs, il importe d'effectuer un bref retour en arrière et de préciser le contexte dans lequel prend place cette élaboration des savoirs. Dans ce but, nous traçons les grandes lignes du mouvement de lutte contre la violence faite aux femmes en Ontario français. Par la suite, nous situons la perspective théorique qui a animé la réflexion autour des enjeux en question, soit le modèle féministe d'élaboration des connaissances utilisé par les intervenantes en VFF, en mettant l'accent sur l'importance de valoriser les connaissances et les expériences qu'elles ont acquises dans l'action.

\section{Grandes lignes du mouvement de lutte contre la VFF en Ontario français}

Pour les besoins de notre analyse, nous divisons l'évolution du mouvement de lutte des femmes francophones de l'Ontario contre laVFF en trois périodes balisées par des événements significatifs : de 1976 à 1993; de 1994 (année du colloque Sensibiliser, décider, agir) à 2004 (États généraux 2004); et de 2005 à 2013 (période où Action ontarienne contre la violence faite aux femmes (AOcVF) s'engage à la préparation de nouveaux États généraux). 
L'année 1976 marque le début des services en français en VFF, à savoir ceux offerts de façon modeste au Ottawa-Hull Rape Crisis Center grâce à la présence d'une intervenante francophone et ceux qu'entraîne l'ouverture de la Maison d'amitié à Ottawa. Rappelons que les premiers services destinés à la majorité linguistique anglophone sont apparus dans le paysage canadien dès 1972. En 1983, deux autres maisons où les femmes peuvent en tout temps avoir accès à des services en français voient le jour, la Maison Interval House à Hawkesbury et Habitat Interlude à Kapuskasing. Quelques programmes de counseling sont offerts en français dans des centres de services familiaux, principalement dans le Nord et dans l'Est de l'Ontario.

En 1988, les intervenantes francophones créent AOcVF. Elles ont besoin de formation et de matériel en français pour elles-mêmes et pour les usagères, puisque très peu des outils et des analyses disponibles dans cette langue tiennent compte de la réalité ontarienne. Les intervenantes ont besoin de participer à un mouvement collectif qui leur ressemble, car dans les regroupements existants, la question des services en français est absente : on ne parle pas, ou que très peu, du défi que soulèvent pour les femmes francophones les lacunes dans l'offre de services en français. Au début des années 1990, poussé à l'action par l'adoption de la Loi sur les services en français, le gouvernement de l'Ontario amorce l'offre de services en français en matière de violence à caractère sexuel.

Débutant en 1994 par un rassemblement des intervenantes lors du colloque Sensibiliser, décider, agir et s'étendant jusqu'à 2004, la deuxième période est marquée par de grands constats entourant les lacunes et le développement de services en français en matière de VFF, et par une volonté collective d'envisager l'avenir et d'étendre de tels services à l'échelle de la province. Les premiers centres d'aide et de lutte contre les agressions sexuelles francophones (CALACS) sont créés. Cette période est aussi marquée par un réveil politique important dans le milieu, et ce, en réaction aux tentatives multiples du gouvernement conservateur ontarien de l'époque d'abolir les CALACS, de limiter de manière importante le mandat des maisons d'hébergement et de s'attaquer aux services 
"Dans le présent article, nous nous intéressons davantage à cette dernière période, parce qu'elle illustre bien les mécanismes de construction collective de développement des connaissances. " en français, notamment par la fermeture préconisée de l'hôpital Montfort. Le sous-financement des services en français en ce qui concerne la VFF se fait sentir partout en province. Les États généraux de novembre 2004 marquent la fin de cette période durant laquelle ont été adoptés de grands principes menant au développement de services en français en matière deVFF (Brunet et Garceau, 2004b).

Fertile en développement de services directs, la troisième période s'étend de 2005 à 2013. Le cap étant maintenu sur la concertation, cette période donne lieu à un approfondissement important des connaissances, à une reconnaissance de la diversité des femmes et des multiples sources d'oppression (grâce à l'analyse féministe intersectionnelle), à des prises de position collectives et à des actions dans le domaine de la VFF, actions entreprises par et pour la communauté des femmes francophones de l'Ontario.

Dans le présent article, nous nous intéressons davantage à cette dernière période, parce qu'elle illustre bien les mécanismes de construction collective de développement des connaissances. Ayant acquis maturité et pérennité, le mouvement et les organismes en VFF peuvent enfin se préoccuper d'enjeux autres que celui de leur propre survie.

Les principaux enjeux qui ont alors animé les débats des intervenantes sont présentés dans une recherche qualitative effectuée pour le compte d'AOcVF en vue des États généraux 2014 (Garceau et Sirois, 2014). C'est en nous basant sur les documents utilisés et développés à cette occasion que nous avons analysé les mécanismes mis en œuvre par les intervenantes du secteur de la VFF pour construire leurs connaissances sur ces enjeux. Soulignons au passage l'esprit de collégialité dont elles ont fait preuve dans un mécanisme de concertation soutenu et alimenté par de nombreux échanges d'information et des discussions. Nous avons examiné leur apport au développement de connaissances sur les enjeux touchant les Franco-Ontariennes et sur leur mise en application dans l'intervention auprès des usagères de leurs organismes respectifs. Enfin, nous avons examiné le rôle des intervenantes dans la mise sur pied des CALACS et des maisons 
"Grâce à ces savoirs, les intervenantes sont davantage outillées pour soutenir les femmes et mieux habilitées à participer et à revendiquer des changements sociaux en vue de mettre fin à la VFF. » d'hébergement pour femmes victimes de violence conjugale, de même que leurs actions sur le plan social, entre autres, dans les communautés et auprès des gouvernements.

Documenter et comprendre la façon dont les intervenantes développent leurs connaissances sur les enjeux en matière de VFF en Ontario nous semble important. Grâce à ces savoirs, les intervenantes sont davantage outillées pour soutenir les femmes et mieux habilitées à participer et à revendiquer des changements sociaux en vue de mettre fin à la VFF.

\section{Considérations méthodologiques}

AOcVF est un regroupement provincial et francophone d'organismes offrant des services aux femmes victimes de violence en Ontario ${ }^{1}$. Son mandat porte sur la prévention de la VFF, sur la formation des intervenantes, sur la création d'outils et sur le développement des services en français.

La recherche qui a suscité la rédaction de cet article a été effectuée par deux chercheuses mandatées par AOcVF pour préparer le document de travail pertinemment intitulé État des lieux sur les agressions à caractère sexuel et la violence conjugale :services en français et enjeux prioritaires dix ans après les États généraux de 2004, en vue des États généraux de novembre 2014 (Garceau et Sirois, 2014). Quelques instruments de collecte de données ont été utilisés pour en développer le contenu².

Depuis 1995,AOcVF rassemble deux fois l'an les intervenantes afin de discuter des enjeux qui les préoccupent. Pour elles, c'est aussi l'occasion de recevoir de la formation et d'échanger des outils. Deux intervenantes représentant chacun des organismes membres et venant de tous les coins de la province participent à chaque rencontre d'une durée de deux jours et demi. De 2005 à 2013, soit la période retenue pour cet article, de trente à cinquante intervenantes ont participé tous les six mois à ces rencontres, dont l'ordre du jour comprenait un tour de table où chacune pouvait 
exprimer les besoins et les défis de son organisme. Les participantes pouvaient y ajouter à l'ordre du jour des sujets dont elles voulaient discuter, ou sur lesquels partager des informations, des idées ou des conseils. Pour les besoins de notre recherche, nous avons procédé à une analyse de contenu thématique de tous les comptes rendus des rencontres bisannuelles des intervenantes des CALACS et des maisons d'hébergement de 2005 à 2013. Nous avons aussi effectué une recension et une analyse de la documentation produite durant cette période et qui porte sur les enjeux liés à la VFF et sur les services en français; nous avons aussi tenu compte des recherches et des documents produits par AOcVF pour la même période ${ }^{3}$.

\section{Le modèle féministe, un modèle savoir et pouvoir de recherche qui défait les oppressions}

«Je me souviens avoir participé à une rencontre à Toronto, vers 1992 ou 1993. Les femmes francophones du réseau m'y ont envoyée en disant "Vas-y! C'est à ton tour!" Näive, j’y suis allée, pour me faire engueuler copieusement quand j'ai osé parler de services en français et constater à quel point nous étions coupées des informations qui circulaient sur les enjeux, les projets de loi, les développements des programmes, etc. À l'époque, nous avions peu d'alliées et nous n'avions pas accès à l'information qui nous permettrait d'avoir le sentiment d'avoir une emprise sur les défis à relever. Je crois que notre réseau était en mode survie, non seulement à cause du financement inadéquat, mais aussi à cause du peu de pouvoir qu'il avait sur l'ensemble des situations qui touchent les femmes : les tribunaux, les programmes, l'accès aux ressources, l'accès aux décideurs, etc. On avait du travail à faire! " (Ghislaine Sirois, entrevue dans Garceau, Savard et Richard, 2012, p. 24) 
"Cette citation

traduit le sentiment

d'oppression qui

s'exerçait alors et

qui s'exerce encore

aujourd'hui contre les

francophones vivant en milieu minoritaire. "

"Appliqué aux enjeux féministes, le slogan "le savoir c'est le pouvoir 》 peut être compris de deux façons : le savoir qui tente de définir ce que sont les femmes dans leurs réalités et le savoir dont elles disposent pour réaliser leurs aspirations. "
Cette citation traduit le sentiment d'oppression qui s'exerçait alors et qui s'exerce encore aujourd'hui contre les francophones vivant en milieu minoritaire. De plus, ces propos soulèvent toute la question de l'occultation des savoirs ou des connaissances qui auraient pu permettre aux groupes francophones de prendre leur place au même titre que leurs homologues anglophones. L'iniquité en la matière était patente. Or, comme le faisait remarquer Waridel (2012, p. 12), « si le savoir est un gisement de pouvoir, comment en assurer le partage équitable? ».

Au début de la deuxième vague du féminisme, certains slogans ont fait école, par exemple "le privé est politique " et "le savoir c'est le pouvoir ". Populaire dans les collectives cherchant à comprendre autrement les réalités féminines, la remise en question du pouvoir sur les femmes et de son lien avec le savoir érigé jusqu'alors en vérité absolue, garante de la domination masculine, a permis à ces dernières de redéfinir les enjeux et d'offrir à la violence que vivent certaines d'entre elles des explications autres que celles qui étaient alors courantes : "La représentation du monde comme le monde lui-même est l'opération des hommes; ils le décrivent du point de vue qui est le leur et qu'ils confondent avec la vérité absolue " (de Beauvoir, 1949, p. 192).

Appliqué aux enjeux féministes, le slogan « le savoir c'est le pouvoir » peut être compris de deux façons :le savoir qui tente de définir ce que sont les femmes dans leurs réalités et le savoir dont elles disposent pour réaliser leurs aspirations. Ces deux facettes sont envisagées ici, et ce, pour deux raisons : d'abord parce que connaitre les réalités des femmes, en particulier dans le domaine de laVFF - laquelle, on le sait, est perpétrée en majorité par des hommes - doit aller au-delà de la représentation traditionnelle qui en est faite; ensuite, parce que les femmes francophones de l'Ontario travaillant dans ce domaine ont développé de solides connaissances sur de multiples problématiques dans le but justement d'acquérir un certain pouvoir, individuel et collectif, leur permettant de passer à l'action. Il s'agit donc pour nous de réhabiliter les expériences des femmes œuvrant en VFF et de les rendre visibles afin d'enrichir et de transformer certains débats et pratiques sociales. 


\section{Les réalités des femmes}

À partir d'une épistémologie féministe, il importe de situer les problématiques en tenant compte du contexte dans lequel elles se manifestent. Comme le note Presley (2003, p. 3), l'épistémologie féministe accorde une crédibilité aux milieux de provenance et aux savoirs des femmes :

Feminist epistemology is concerned with « whose knowledge " is being considered. Feminist epistemologists critique traditional epistemology and argue for ways of understanding knowledge that focus on context and situation. Feminist epistemologists do not suggest that empirical evidence is wrong, but rather that it is necessary to understand that most beliefs are as much a result of their social context as they are factually true.

Les réalités des femmes doivent se comprendre du point de vue des femmes qui vivent la violence, en d'autres mots dans la production de « savoirs situés » (Puig de la Bellacasa, 2003). Ce sont ces multiples réalités qui sont partagées par les intervenantes tout au long des rencontres. En tant que sujets et actrices sociales, les intervenantes et les femmes violentées doivent être consultées et entendues, car elles sont des informatrices clés sur les réalités qu'elles sont les premières à vivre et sur les solutions qu'elles mettent en œuvre, des solutions adaptées (Risse et Smedslund, 2014). En effet, en rapportant et en partageant l'expérience vécue par les femmes qui utilisent en région les services communautaires ou institutionnels et qui privilégient les services en français, les intervenantes enVFF en Ontario français sont les yeux et la voix des femmes francophones.

En recherche féministe, les femmes sont encouragées à raconter leurs histoires et leurs situations. Ce faisant, elles sont en mesure de contester les experts et les idéologies dominantes qui les oppriment en limitant leurs connaissances et leur compréhension 
de la réalité (Liamputtong, 2007). Ainsi, le développement de connaissances par les intervenantes s'inscrit dans la recherche féministe et s'exprime lors de rencontres organisées expressément pour elles. Ces rencontres leur permettent de raconter sans contraintes des réalités vécues par des femmes et légitiment de façon respectueuse leurs voix comme source de connaissances (Liamputtong, 2007). Il est vrai qu'ici ces réalités sont filtrées par l'interprétation qu'en font les intervenantes. Mais ces dernières rapportent à chaque rencontre autant les possibilités que les limites des systèmes mis en place pour soutenir les femmes aux prises avec la violence. Examinés sur une période de dix ans grâce aux comptes rendus des rencontres - qui sont aussi en soi un filtre additionnel -, les propos des intervenantes peignent un portrait assez juste des défis rencontrés au niveau provincial.

La participation à l'élaboration des connaissances aide à se défaire de l'oppression (Khanlou et Peter, 2005, cité dans Liamputtong, 2007). Dans le cas des femmes d'expression française en Ontario, en plus de remettre en cause les privilèges masculins, elles ont aussi eu à se questionner sur les privilèges de la communauté linguistique dominante, privilèges qui peuvent aisément se transformer en oppression. De plus, les intervenantes sont constamment aux prises avec les difficultés typiques de faire valoir un savoir minorisé afin de forcer les savoirs établis à en tenir compte. Par ailleurs, avec l'avènement de l'analyse féministe intersectionnelle, on reconnait désormais que les femmes ne forment pas un groupe homogène et que le pouvoir n'est pas réparti de façon égale selon les caractéristiques personnelles et collectives des femmes, par exemple, la race, l'orientation sexuelle ou l'appartenance à une communauté minoritaire ou majoritaire.

La recherche féministe participative a pour but d'aider les personnes à se remettre et à se libérer des contraintes imposées par des structures sociales irrationnelles, improductives, injustes et insatisfaisantes, qui les limitent dans leur autodéveloppement et leur autodétermination (Khanlou et Peter 2005, cité dans Liamputtong, 2007, [notre traduction]). Nous référant à ce type de recherche, nous pouvons affirmer que les intervenantes francophones ont pris part à un processus d'inspiration féministe 
de développement des connaissances qui avait pour but de défaire l'oppression vécue comme femmes francophones.

De la même manière, Brydon-Miller (2001, p. 80, [notre traduction]) propose que la recherche féministe repose sur trois principes fondamentaux :

Elle met l'accent sur les individus, les groupes et les communautés qui ont toujours été opprimés ou exploités.

Elle tente d'identifier à la fois les préoccupations particulières des populations locales et l'origine principale des clés de leur oppression et elle vise à parvenir à un changement social positif.

Il s'agit d'une forme de recherche, d'éducation et d'action qui exige que toutes les participantes apportent leurs connaissances et leurs compétences particulières, et à travers cette contribution, toutes les participantes acquièrent de nouvelles connaissances.

Le processus suivi par les intervenantes ayant participé aux rencontres bisannuelles s'inscrit donc dans une forme de recherche qui adapte un modèle féministe et qui y adhère.

\section{Prendre part à l'action en vue de changement social}

Depuis plus de trente ans, les actrices du mouvement des femmes, notamment en Amérique du Nord, se mobilisent pour mettre en œuvre de nouvelles pratiques d'intervention qui s'inscrivent dans une perspective non seulement d'ouverture, de respect et d'écoute à l'égard des besoins exprimés et des expériences vécues par les femmes, mais encore de changement individuel et social. (Corbeil et Marchand, 2010, p. 23)

Depuis ses débuts, AOcVF et ses groupes membres, inspirés par le partage des expériences et l'analyse faite des enjeux rencontrés au quotidien, suivent intuitivement un processus qui 
correspond au cadre de la coconstruction des savoirs comprenant les quatre axes suivants : l'observation, la réflexion, la planification et l'action (Équipe Praxit, 2011). En effet, les rencontres bisannuelles des intervenantes, dont les comptes rendus constituent l'une de nos sources principales de collecte de données, ont toujours été l'occasion de partager le vécu des femmes (l'observation), vu à travers l'expérience des intervenantes. Leurs savoirs et ceux des usagères qu'elles partagent sont des savoirs inestimables, à partir desquels il est possible de dégager des enjeux, de constituer des analyses (la réflexion), de planifier des actions (la planification) et de les mettre en œuvre (l'action).

Liamputtong (2007, p. 14) propose une démarche similaire, quoique dans un ordre différent, dans sa description du processus de recherche féministe. Il ajoute que la connaissance doit redonner du pouvoir aux femmes, qu'elles soient chercheuses ou sujets de recherche :

Feminist research focuses on gender and gender inequality and this implies a strong moral and political commitment to decreasing inequality;

It aims to give voice to personal and everyday experiences of women and other marginalised individuals and groups;

It commits to social activism and aims to bring about social transformation which improves the situation and conditions under which women and the marginalised live;

It promotes reflexivity which critically scrutinises how social structures like gender, ethnicity, social class, and sexual orientation as well as larger social, economic and political conditions of the researchers may impact on the research process; and

It rejects the traditional imbalanced power relationships between the researcher and the 
researched and attempts to provide the research participants with more power in the research enterprise.

Nous avançons que le processus suivi par les intervenantes correspond à une approche structurée, même si elle a été surtout intuitive dans la pratique, et basée sur des principes d'intervention féministe. La compréhension de ce processus pourrait servir à poursuivre et arrimer la pratique au besoin.

\section{Les enjeux liés à la VFF}

"Ayant créé de nouveaux services, il était devenu nécessaire pour elles d'approfondir les divers enjeux issus des milieux de pratique, et ce, tant pour donner une voix aux femmes qui utilisent leurs services que pour répondre à leurs besoins, et ensemble, chercher à opérer des changements sociaux propres à améliorer une situation problématique. "
Au cours de la dernière décennie, en plus de continuer à revendiquer pour améliorer l'accès aux services en français en matière de VFF, les intervenantes francophones du réseau d'AOcVF ont misé sur le développement de connaissances liées à de très nombreuses problématiques s'y rattachant (Garceau et Sirois, 2014). Ayant créé de nouveaux services, il était devenu nécessaire pour elles d'approfondir les divers enjeux issus des milieux de pratique, et ce, tant pour donner une voix aux femmes qui utilisent leurs services que pour répondre à leurs besoins, et ensemble, chercher à opérer des changements sociaux propres à améliorer une situation problématique. Comme ces enjeux sont nombreux, nous utilisons ici deux cas de figure pour démontrer la construction des savoirs par les intervenantes. Le premier enjeu relève plus particulièrement, mais non exclusivement, du domaine de la violence à caractère sexuel, soit la prostitution. Le deuxième enjeu porte sur l'accès aux services juridiques lorsqu'une femme est aux prises avec la violence conjugale. Pour chacun de ces thèmes, nous tentons de démontrer les étapes d'observation, de réflexion, de planification et d'action. Par la suite, nous verrons si le processus s'apparente à la démarche proposée par Liamputtong (2007). 


\section{Deux cas de figure}

La prostitution et l'accès aux services juridiques en droit de la famille pour les femmes aux prises avec de la violence ont fait l'objet de discussions intenses et d'échanges d'information à presque chacune des rencontres des intervenantes du secteur de la VFF en Ontario français entre 2005 et 2013. Ces deux enjeux permettent de voir comment les intervenantes ont développé leurs connaissances et les ont transposées dans l'action.

\section{La prostitution}

Le thème de la prostitution faisait l'objet de discussions chez les intervenantes bien avant la période choisie comme objet d'étude. Toutefois, en 2005, le sujet revient à l'avant-scène de l'actualité alors qu'un comité parlementaire de la Chambre des communes s'affaire à rédiger un rapport sur la prostitution au Canada (Comité permanent de la justice et des droits de la personne, 2006). Le sujet attire l'attention du public et les médias en parlent abondamment. Les intervenantes se sentent interpellées, d'autant plus que le nombre des usagères victimes de la prostitution va en augmentant dans certains organismes du réseau de laVFF. En mai 2005, un groupe d'intervenantes participe à Vancouver à une rencontre de l'Association nationale Femmes et Droit (ANFD) et du Legal Education and Action Fund (LEAF) de la côte Ouest. Divers ateliers portant sur la prostitution y sont présentés, dont l'un portant sur le modèle suédois qui criminalise les proxénètes et les clients, mais pas les personnes prostituées.

La même année, une conférence réunit d'autres intervenantes à Montréal; l'idée de faire du sexe un travail comme les autres y est soulevée. Les intervenantes constatent que les éléments de discussion recoupent ceux d'une étude récente de chercheuses de l'Université d'Ottawa (Bruckert, Parent et Pouliot, 2006) en collaboration avec le Centre Espoir Sophie d'Ottawa.

Toutes ces informations sont ramenées par les intervenantes lors de la rencontre bisannuelle suivante où deux intervenantes 
du Nord de l'Ontario témoignent alors du travail qu'elles font auprès des femmes prostituées et des danseuses exotiques dans leur région. La discussion s'entame... Deux sites internet d'information, Sisyphe et netfemmes, ayant des positions diamétralement opposées, sont proposés aux intervenantes à des fins de consultation et de discussion. Pour répondre aux souhaits exprimés par les intervenantes de poursuivre la réflexion sur le sujet,AOcVF soumet à Condition féminine Canada une demande de subvention visant à approfondir la recherche dans ce dossier.

En 2006, les deux mêmes intervenantes du Nord de la province exposent aux autres intervenantes leur projet d'intervention auprès des danseuses exotiques. Intitulé Powerline, il a pour objectif de fournir des ressources aux danseuses exotiques afin qu'elles puissent recourir à des services en cas de besoin. Elles partagent avec le groupe des informations sur les danseuses exotiques : les trois quarts d'entre elles sont francophones, elles proviennent pour la plupart du Québec, elles sont enrôlées dans un réseau itinérant de danseuses qui fait le circuit des bars québécois et ontariens. Plusieurs d'entre elles sont aux prises avec des problèmes de toxicomanie et vivent des relations violentes. Pourtant, certaines intervenantes mentionnent que les femmes prostituées parlent souvent de liberté et de vivre leur sensualité. À cela, une autre intervenante rapporte que l'organisme Stella de Montréal, qui se veut le défenseur des travailleuses du sexe, «normalise le travail du sexe et parle peu de la violence ".

En 2007, AOcVF indique aux intervenantes réunies à la rencontre bisannuelle qu'une analyse des droits humains des femmes en regard de la prostitution a été amorcée en réponse aux préoccupations formulées. Pour ce faire, les services de la spécialiste en droits des femmes, Shelagh Day ${ }^{4}$ ont été retenus. Au Québec, comme en témoignent les informations sur les sites Web de l'époque, le débat est enflammé, mais il semble susciter peu d'émois en Ontario. Lors de cette même rencontre, une intervenante en provenance d'un CALACS informe le groupe que dorénavant son centre n'utilisera plus le vocable «travailleuse du sexe ", ne voulant plus perpétuer la notion que c'est un travail comme un autre. L'ensemble des intervenantes adoptera cette 
pratique et parlera dorénavant de « femmes prostituées » ou de "femmes prises dans la prostitution".

En 2008, le rapport de Shelagh Day, La prostitution, une violation des droits humains des femmes pauvres, est présenté lors d'une rencontre. Les intervenantes présentes en entérinent les recommandations, s'inscrivant ainsi dans la ligne abolitionniste en regard de la prostitution, en opposition à la légalisation totale du commerce du sexe. Lors de la même rencontre, des discussions portent sur l'hypersexualisation des jeunes filles avec le visionnement de Sexy Inc. Nos enfants sous influence de Sophie Bissonnette (ONF,2007) et la présentation des ouvrages Le sexe en mal d'amour de Jocelyne Robert (2005) et La sexualisation précoce des filles de Pierrette Bouchard, Natasha Bouchard et Isabelle Boily (2005). Le trafic humain est également à l'ordre du jour, ce trafic ayant un lien important avec l'exploitation sexuelle des femmes et des jeunes.

En 2009, les groupes membres d'AOcVF organisent des ateliers sur l'exploitation sexuelle dans les régions de l'Ontario. AOcVF développe alors un webinaire permettant aux intervenantes de poursuivre leurs discussions et distribue des articles sur le risque encouru par les filles de tous les milieux, sur les clients comme premiers agents de la prostitution et sur la démarche que ces derniers devraient entreprendre quant au passage de "clients " à celui d'" hommes responsables » de leur sexualité, recherchant le consentement et des rapports égalitaires entre partenaires. Lors des rencontres, les intervenantes accèdent aux sites où sont débattues les diverses positions de légalisation, de décriminalisation ou d'encadrement juridique selon le modèle suédois. Elles visionnent la vidéo Not for Sale/Pas à vendre de Marie Vermeiren (2006). Les discussions se poursuivent.

En 2010, le sujet est toujours à l'ordre du jour, car c'est à ce moment que la Cour supérieure de l'Ontario rend sa décision dans la cause Bedford c. Canada; le jugement Himel invalide les trois dispositions du Code criminel concernant la prostitution, à savoir l'interdiction du proxénétisme sauf s'il est "forcé " (le fardeau de la preuve reposant sur la victime), la sollicitation des clients dans la rue et la tenue de maisons closes. Pour la Coalition 
des femmes pour l'abolition de la prostitution ${ }^{5}$ dont fait partie AOcVF, cette décision ne tient nullement compte des inégalités entre les hommes et les femmes et se soucie plus d'ordre public que des droits à la sécurité des femmes.

En 2011, la Coalition demande le statut d'intervenant devant la Cour d'appel de l'Ontario et prépare un mémoire ${ }^{6}$ qui propose la criminalisation asymétrique de la prostitution, c'est-à-dire de ne pas punir les femmes prises dans la prostitution, mais de pénaliser l'exploitation des femmes par les clients prostitueurs et les proxénètes. Les intervenantes expriment le souhait qu'un mapping soit fait de la situation de la prostitution en Ontario, comme celui mené à Montréal par la Concertation des luttes contre l'exploitation sexuelle (CLES), un organisme abolitionniste très actif dans le débat sur la prostitution et aussi membre de la Coalition pancanadienne. La CLES offre aux intervenantes une formation de deux jours qui a pour but de soutenir les intervenantes qui désirent faire de l'éducation publique sur la prostitution. Lors de cette même rencontre, le film L'imposture d'Ève Lamont (2010) est présenté aux intervenantes, de même que Je vous salue... Le point zéro de la prostitution, un ouvrage de Rose Dufour (2005). Les intervenantes assistent aussi à une présentation de la Gendarmerie Royale du Canada sur le trafic humain. Plusieurs intervenantes étaient avides d'information après avoir rencontré des femmes victimes d'un tel trafic.

En 2012, la Coalition pancanadienne des femmes pour l'abolition de la prostitution obtient le statut d'intervenant auprès de la Cour Suprême du Canada. En juin 2013, elle y soumet un mémoire soutenant la thèse abolitionniste. À titre de membres de la Coalition par le biais d'AOcVF, les intervenantes des services en français enVFF en Ontario ont été consultées sur les principaux arguments du mémoire. Selon ce dernier, la prostitution doit d'abord être perçue comme une question d'inégalité entre les hommes et les femmes et le législateur doit assurer les droits des femmes à la sécurité, en particulier ceux des femmes autochtones souvent exploitées dans le système prostitutionnel à cause du racisme, de la pauvreté et de la colonisation. Le mémoire propose à nouveau la criminalisation asymétrique de la 
"Confiantes, grâce aux présentations et aux discussions, les intervenantes étaient prêtes à participer à des débats, à offrir des ateliers dans leurs communautés ou à participer à des entrevues dans les médias. " prostitution. De plus, les intervenantes participent à des journées de formation sur les arguments à invoquer et les diverses stratégies à favoriser au moment où la Cour suprême se prononcera sur la constitutionnalité des articles portant sur la prostitution dans le Code criminel canadien. La décision rendue par la Cour suprême le 20 décembre 2013 a effectivement invalidé les dispositions de la loi actuelle et accordé une année au gouvernement fédéral pour agir s'il ne voulait pas que la prostitution au Canada soit, de facto, presque entièrement légalisée ${ }^{7}$.

Confiantes, grâce aux présentations et aux discussions, les intervenantes étaient prêtes à participer à des débats, à offrir des ateliers dans leurs communautés ou à participer à des entrevues dans les médias. Elles ont aussi discuté des mesures de sécurité élaborées par l'Association nationale des femmes autochtones et visionné une vidéo du groupe Stop The Traffik ${ }^{8}$, dont l'objectif est de mettre fin au trafic humain. Parallèlement, les intervenantes ont appris l'existence de l'Adult Entertainment Association ${ }^{9}$ qui n'attend que le feu vert pour ouvrir des maisons de débauche pour lesquelles elle entend recruter - avec tambours et trompettes des jeunes femmes sur les campus canadiens.

\section{Accès aux services juridiques}

Dans cette section, nous verrons comment les intervenantes ont construit leurs connaissances sur la question de l'accès aux services juridiques en droit de la famille et comment elles ont mis en action ces connaissances afin d'obtenir de meilleurs services pour les femmes francophones aux prises avec de la violence en Ontario. Mais, auparavant, il importe de regarder le contexte de l'accès aux services en français en matière de droit de la famille.

Depuis 1994, lors des rencontres bisannuelles réunissant les intervenantes, ces dernières font état du peu d'accès qu'ont les femmes victimes de violence à des services juridiques, plus particulièrement en matière de litige civil et en droit de la famille. C'est toujours le cas en 2005 et en 2006. Dans certaines régions de la province, le bassin d'avocates et d'avocats pratiquant le droit familial en français est si restreint, se limitant souvent à une 
seule ou à un seul avocat, que dans une dispute juridique entre conjoints, si l'un des membres retient les services de l'unique avocat pratiquant le droit de la famille dans la région, l'autre n'y aura pas accès. Une telle situation n'est pas rare et s'aggrave, surtout si l'on songe que pour la conjointe, en raison de ses faibles revenus, il lui faut préalablement détenir un certificat de l'aide juridique pour obtenir des services. Entre temps, il y a de fortes possibilités de penser que le conjoint aura déjà retenu les services de l'avocate ou l'avocat en question, ne serait-ce qu'en raison de son revenu supérieur.

En 2007, des intervenantes indiquent qu'elles doivent servir d'interprètes pour une usagère lors de visites chez une avocate ou un avocat anglophone. Ce genre d'accompagnement n'est pas sans risque pour l'usagère ou pour l'intervenante, car peut alors se produire une erreur d'interprétation ou de compréhension. Mais peut-on éviter une telle situation dans une région où l'usagère ne peut être servie en français faute de services juridiques dans cette langue? En 2008, dans la région du Nord-est, les usagères devaient faire plus de deux heures de route pour rencontrer une avocate ou un avocat. Toutefois, dans l'Est ontarien, on indique que l'accès à des avocates ou avocats en droit de la famille est plus facile. Cette région de la province fait figure d'exception, puisque du Nord au Sud, les intervenantes déplorent toujours le manque d'accès à des services juridiques en français.

L'accès à des services juridiques n'est pas un problème exclusif aux femmes d'expression française, c'est un problème généralisé, d'abord pour des questions de coûts :

Dans son rapport en 2013, le Comité d'action sur l'accès à la justice sociale en matière civile et familiale a conclu que le problème d'accès à la justice, et en particulier en droit de la famille, est "grave et urgent ". Les coûts peuvent atteindre entre $13000 \$$ et $37000 \$$ pour un procès de deux jours, ce qui est inabordable pour la majorité des Canadiennes. (Garceau et Sirois, 2014, p. 130) 
Ce problème est aussi aggravé par le manque de financement accordé en vertu du régime de l'aide juridique. Celui-ci a normalement pour but de rendre accessibles les services juridiques en droit de la famille pour les personnes qui n'en ont pas les moyens. Or, dans les faits,

le gouvernement fédéral alloue [aux provinces] des fonds ciblés directement pour soutenir l'aide juridique en droit pénal. Aucun financement fédéral semblable (sic) [n'est] voué à l'aide juridique civile. Les provinces ont plutôt le choix, à leur discrétion, de financer l'aide juridique dans les affaires civiles à partir du Transfert canadien en matière de programmes sociaux. (Alliance canadienne féministe pour l'action internationale, 2008, p. 22)

Les gouvernements provinciaux préferent s'en tenir le plus possible au droit pénal plutôt qu'au droit de la famille. Le recours à l'aide juridique est toujours difficile, souvent à cause des barèmes d'admissibilité établis en fonction d'un revenu très bas. De plus, la plupart des cliniques juridiques communautaires n'offrent pas de services en droit de la famille, sauf à Toronto où se trouve une clinique spécialisée en droit de la famille pour les femmes aux prises avec la violence, le Barbara Schliffer Commemorative Legal Clinic. Nous ne pouvons pas nous empêcher de noter une forme de discrimination basée sur le sexe en matière d'aide de services juridiques. En effet, lorsque les hommes ont besoin de services juridiques, il s'agit le plus souvent pour des causes liées à des délits criminels. Lorsque les femmes en ont besoin, il s'agit de causes liées au droit de la famille :

Les gouvernements fédéral et provinciaux justifient la préférence accordée à l'aide juridique en droit pénal au motif que des questions fondamentales de liberté sont en jeu dans ces causes. Dans les affaires au civil qui sont souvent le lot des femmes, des atteintes tout aussi graves à leurs droits ne sont pas reconnues comme telles, 
même si les femmes y perdent leur sécurité et leur capacité de jouir de leurs droits sociaux et économiques. (Alliance canadienne féministe pour l'action internationale, 2008, p. 23)

Il s'agit donc d'une forme de discrimination systémique. Lorsqu'on considère le revenu annuel moyen des femmes (31 100 \$) et celui des hommes (45 200 \$) (Canadian Centre for Policy Alternatives, 2012), il nous apparaît évident que l'insécurité économique guette les femmes si elles ne peuvent faire valoir leurs droits au tribunal de la famille ${ }^{10}$, ce qu'elles peuvent difficilement se permettre. En effet, celles qui mettent fin à une relation violente utiliseraient une banque alimentaire vingt fois plus souvent que la moyenne des utilisateurs de telles ressources, et ce, sur une période pouvant s'étendre jusqu'à trois ans (McInturff, 2013). Grâce au lobby des groupes de femmes, on constate qu'il y a eu des améliorations depuis quelques années avec l'instauration d'une ligne téléphonique d'Aide juridique Ontario et la présence d'une ou d'un avocat de service au Bureau d'information juridique (FLIC) à la cour. Cependant, ces nouveaux services ne répondent que partiellement aux grands besoins des femmes aux prises avec des questions de garde légale ou de pensions alimentaires dans un cas de violence conjugale. Voyons maintenant comment les intervenantes ont bâti leurs connaissances à ce sujet.

En plus de l'insécurité économique vécue par les femmes aux prises avec de la violence, les intervenantes sont très conscientes de la réelle menace que présente le conjoint, car souvent la violence s'aggrave au moment de la séparation. Selon elles, les ex-conjoints font usage de tactiques abusives pour continuer à exercer leur pouvoir et de garder le contrôle sur leurs ex-partenaires. Par exemple, un ex-conjoint peut choisir un procès qui se déroule en anglais lorsqu'il est accusé en droit pénal, sachant que la conjointe ne pourra suivre les débats. Cela dit, c'est en cherchant à obtenir la garde légale (entière ou partagée) des enfants que se manifeste davantage le désir de garder le contrôle sur la mère et d'éviter ainsi de payer une pension alimentaire. Ce n'est pas une problématique nouvelle, les intervenantes en parlent déjà depuis plusieurs années, 
"Romito (1997) fait état de quatre stratégies utilisées afin de contrer les tentatives des groupes féministes de faire valoir les droits à l'égalité, quatre stratégies bien utilisées dans ce débat..." et surtout depuis que le gouvernement fédéral envisage une réforme de la Loi sur le divorce, avec au cœur des débats la garde légale des enfants et les pensions alimentaires.

Les intervenantes reconnaissent les défis que doivent affronter les femmes et voient comment s'organise le backlash du mouvement masculiniste à leur endroit. Romito (1997) fait état de quatre stratégies utilisées afin de contrer les tentatives des groupes féministes de faire valoir les droits à l'égalité, quatre stratégies bien utilisées dans ce débat :

1. la reformulation de la problématique (droits des pères);

2. la psychologisation de la problématique (syndrome de l'aliénation parentale);

3. la "biologisation dure ", ce qui dans ce cas pourrait être le rôle " traditionnel " et biologique des pères et des mères;

4. l'utilisation de termes vagues, comme "violence familiale " plutôt que «violence faite aux femmes » ou «VFF ».

Les intervenantes sont conscientes de telles stratégies et les incorporent à leurs analyses.

Des groupes comme Fathers for Justice investissent les bureaux des députés et ministres à Ottawa. Ils ont une rhétorique parfois extrême et l'oreille de certains députés. Les intervenantes décident de montrer publiquement à quels discours les femmes sont confrontées, mais celles qui sont présentes dans les médias sont parfois prises à partie. Les rencontres bisannuelles leur permettent d'améliorer leur argumentaire et réconfortent celles qui ont été exposées à des confrontations médiatisées.

En 2005, un lobby de groupes religieux cherche à obtenir que les règles régissant les familles dans leur contexte religieux aient un statut juridique égal à celui du droit de la famille ontarien. Cette revendication est mieux connue pour avoir proposé la création d'un tribunal de la Charia en Ontario, chargé de rendre justice dans des causes relevant du droit de la famille. Or, on l'oublie souvent, ce ne sont pas que des représentants de la religion musulmane qui sont partie prenante dans cette revendication; des lobbys juifs et chrétiens sont aussi impliqués. Dès lors, les intervenantes s'organisent devant ce qu'elles considèrent être 
une forme de privatisation des droits des femmes : des droits publics sont ainsi renvoyés à la sphère privée, la pratique de la religion et la confessionnalité étant du domaine privé dans nos démocraties libérales. Inquiètes que les femmes soient soumises à des diktats religieux ne respectant pas leurs droits de citoyennes, les intervenantes participent à une campagne d'information, de lettres et elles obtiennent des rencontres avec leurs députées et députés et les ministres concernés. En solidarité avec de multiples associations, les intervenantes demandent qu'il n'y ait qu' "un seul droit de famille " pour toutes les Ontariennes et que les femmes n'aient pas à renoncer à leurs droits citoyens en vertu de leur appartenance religieuse. Le premier ministre de l'époque, Dalton McGuinty, annonce alors que son gouvernement décline les revendications des groupes religieux. Il annonce de plus le financement d'une vaste campagne d'information juridique en droit de la famille spécifiquement à l'intention des femmes qui sous l'influence de leaders religieux pourraient être mal informées sur leurs droits. La campagne Femmes ontariennes et droit de la famille, menée en français par AOcVF, produit dès 2007 des dépliants à l'intention de toutes les femmes. Des dépliants destinés aux femmes musulmanes et juives sont aussi produits en partenariat et distribués en français et dans plusieurs autres langues, dont certaines sont parlées dans des pays de la francophonie, par exemple, en créole et en lingala. Les intervenantes ont en main quantité d'outils pour informer les femmes de leurs droits en matière de droit de la famille.

Un autre aspect juridique important soulevé par les usagères et les intervenantes touche la question des contre-accusations. À la suite de l'adoption de politiques de tolérance zéro en matière de violence, on constate que les policières et policiers appelés à intervenir dans une situation de violence domestique déposent de plus en plus d'accusations de voies de fait, et ce, tant à l'égard des conjointes que des conjoints, sans chercher à savoir si les gestes posés par les femmes étaient de légitime défense ou du moins de nature défensive. En conséquence, de plus en plus de femmes se voient accusées de voies de fait :

Les intervenantes des maisons d'hébergement disent que, dans de tels cas, les femmes accusées 
plaident rapidement et souvent coupables, question d'en finir avec le système de justice et de pouvoir retourner à la maison s'occuper des enfants. Toutefois cette pratique a des conséquences négatives, car dorénavant ces femmes ont un dossier criminel. Cela pourrait leur nuire notamment lors de l'évaluation en matière de garde des enfants. (Garceau et Sirois, 2014, p. 132)

Par ailleurs, dans la plupart des régions de l'Ontario, on sait que des groupes d'intervention auprès des partenaires violents ont été mis sur pied et que les femmes accusées doivent s'y intégrer sans distinction de genre.

Les femmes ainsi accusées, même lorsqu'elles sont victimes et n'ont fait que se défendre, sont parfois orientées par les tribunaux spécialisés vers les programmes pour hommes violents comme les programmes d'intervention auprès des partenaires violents (PIPV ou PAR, en anglais), qui ne suffisent pas à la demande à plusieurs endroits. Or, si dans certaines régions, ces programmes ont su développer une approche plus sophistiquée allant plus loin que la simple

«En 2008, les intervenantes rapportent que le "syndrome de l'aliénation parentale" est de plus en plus invoqué à l'encontre des femmes, qui supposément feraient tout en leur pouvoir pour convaincre les enfants de ne pas voir leur père." gestion de la colère et qu'ils ont adapté leur intervention à la réalité des femmes victimes de violence, ce n'est pas toujours le cas. (Garceau et Sirois, 2014, p. 132)

Cela dit, dans certaines régions, on crée des groupes pour femmes seulement et on adapte l'intervention en conséquence, car on constate que souvent les femmes qui sont obligées de participer à ces sessions sont de prime abord victimes de violence conjugale.

En 2008, les intervenantes rapportent que le «syndrome de l'aliénation parentale " est de plus en plus invoqué à l'encontre des femmes, qui supposément feraient tout en leur pouvoir pour convaincre les enfants de ne pas voir leur père. Toutefois, ce que les intervenantes constatent c'est que trop souvent ce sont les 
différents acteurs du système de justice qui ne prennent pas en considération la violence du père à l'égard de la mère ou à l'égard de leurs enfants, ce qui pourrait expliquer que la mère ne souhaite pas faciliter le contact afin de protéger ses enfants. Finalement, les intervenantes déplorent le manque de communication entre la cour de la famille et la cour criminelle, en particulier lorsqu'il y a des ordonnances de non-contact entre les ex-conjoints.

Plusieurs occasions d'échanges et de discussion avec des avocates ou des chercheuses communautaires sont organisées dans le cadre des rencontres des intervenantes afin de peaufiner leurs analyses sur les droits des femmes et le droit de la famille :

- En 2006, la directrice générale de l'Association des juristes d'expression française (AJEFO) rencontre les intervenantes pour entendre les difficultés auxquelles font face les usagères des services, dont celle de trouver en régions des avocates ou avocats francophones spécialisés en droit de la famille et en droit de l'immigration. Elle fournit des informations et son soutien politique en faveur de l'obtention d'un meilleur accès à des services en droit de la famille pour les femmes victimes de violence;

- En 2007, la juriste Andrée Côté de l'Association nationale femmes et droit s'adresse aux intervenantes pour discuter avec elles des changements législatifs en regard des droits des femmes à l'égalité;

- En 2009, Julie Lassonde, avocate chercheuse pour le compte de la Commission du droit de l'Ontario, consulte les intervenantes dans le cadre d'une recherche qui vise à mieux répondre aux besoins des femmes victimes de violence dans le processus de justice familiale;

- En 2010, la chercheuse communautaire Lucie Brunet anime une session de consultation sur les besoins juridiques des femmes dans le cadre d'une recherche financée par Aide juridique Ontario (Brunet, Sherwood et Garceau, 2009). Les intervenantes discutent des expériences, des défis et des obstacles vécus par les femmes francophones dans le système juridique et nomment les enjeux et besoins juridiques auxquels il est urgent de répondre; 
"Les échanges d'information et les analyses qu'elles font conjointement, les présentations et les consultations des juristes et des chercheuses contribuent au développement de leurs connaissances dans le domaine. »
- La même année, la chercheuse communautaire Johanne Ouimette, qui participe au Comité consultatif provincial sur la réforme du droit de la famille, présente aux intervenantes les différents enjeux de cette réforme et suscite un échange d'information.

Toutes les occasions que nous venons d'énumérer sont autant de moments formatifs pour les intervenantes. Les échanges d'information et les analyses qu'elles font conjointement, les présentations et les consultations des juristes et des chercheuses contribuent au développement de leurs connaissances dans le domaine. De plus, depuis 2010, AOcVF a embauché une avocate dans le cadre du projet Femmes ontariennes et droit de la famille. Cette dernière peut offrir de l'information à l'occasion de rencontres avec les intervenantes et plus spécialement des formations en droit de la famille et sur des enjeux juridiques en matière d'agression à caractère sexuel. Nous avons vu plus haut que le projet Femmes ontariennes et droit de la famille avait suscité la création de nombreux outils qui sont tous présentés aux intervenantes lors des rencontres. S'ajoutent d'autres outils tels que le Cahier d'information sur le droit de la famille, Après la rupture ou Mieux comprendre la loi, guide pour les femmes ontariennes.

Jusqu'à maintenant, nous avons constaté que les intervenantes ont écrit des lettres, participé à des campagnes d'information et de sensibilisation, rencontré des députées ou députés et des ministres pour discuter du meilleur soutien à offrir aux femmes aux prises avec de la violence en matière de droit de la famille. En 2009, pour pallier les difficultés constatées par les intervenantes, cellesci appuient AOcVF lorsque l'organisme dépose un projet de développement de services juridiques en droit de la famille. Inspiré des services offerts par le Barbara Schliffer Commemorative Legal Clinic, le modèle proposé aux femmes francophones est toutefois «hors murs ». En effet, il propose l'utilisation de nouvelles technologies des communications dans l'offre des services afin qu'ils soient accessibles aux femmes dans toutes les régions de l'Ontario. Ces services offrent aux femmes francophones victimes de violence de l'information et du soutien dans leurs démarches. Ils sont offerts en collaboration avec les organismes situés dans 
les régions, tels que les maisons d'hébergement. Depuis 2012, ces services, quoique très partiels, sont maintenant en place.

\section{Conclusion}

"Le processus que nous avons décrit renforce les capacités du secteur de la VFF en Ontario français, un secteur marqué par l'approche féministe, communautaire et participative afin qu'il soit en mesure de mettre en cuvre l'un de ses principes de gouvernance, soit le " par et pour " les femmes francophones. "
Dans cet article, nous avons décrit la manière dont les intervenantes du secteur de laVFF ont travaillé collectivement afin de développer des connaissances sur les enjeux qui y sont liés. Nous avons utilisé le modèle "observation, réflexion, planification et action " (Équipe Praxit, 2011) pour montrer comment ces intervenantes ont réussi à transposer un vaste ensemble de connaissances dans leurs rapports avec des usagères des CALACS et des maisons d'hébergement pour femmes victimes de violence conjugale, d'où émanait la demande initiale. Nous avons montré la mise en place de revendications pour des changements sociaux que ce soit à travers des outils de sensibilisation, des tournées d'informations dans les écoles, la signature de pétitions ou la rencontre avec des députées ou députés.

La façon d'aborder l'acquisition de connaissances telle que pratiquée par $\mathrm{AOcVF}$ et ses groupes membres constitue un acte féministe et s'inscrit dans l'approche favorisée par Liamputtong (2007) en ce qui a trait à l'analyse de genre et des inégalités des défis vécus au quotidien, en contexte, tout en favorisant le partage des savoirs, donc du pouvoir, entre toutes les intervenantes.

Le processus que nous avons décrit renforce les capacités du secteur de la VFF en Ontario français, un secteur marqué par l'approche féministe, communautaire et participative afin qu'il soit en mesure de mettre en ouvre l'un de ses principes de gouvernance, soit le " par et pour» les femmes francophones. En effet, si le secteur ne pouvait se prévaloir de connaissances solides en matière deVFF, il ne serait pas en mesure d'offrir des services de qualité en français à travers la province.

La concertation et la pratique de discuter deux fois l'an des défis rencontrés ont permis aux intervenantes d'observer 
les réalités complexes de la VFF et les nombreux, et tout aussi complexes, besoins des femmes aux prises avec cette violence. Elles ont aussi contribué à mettre en commun ces réalités et ces besoins et d'aboutir à une réflexion collective qui permet aux intervenantes de participer à l'élaboration de solutions et de les transposer en actions pour mieux répondre aux besoins des femmes aux prises avec de la violence. Les intervenantes en VFF sont des analystes et des actrices sociales qui ont développé une analyse critique des problèmes vécus par les femmes. Leur discours vient contrecarrer un certain discours ambiant selon

"...elle permet de donner la parole à celles qui sont souvent ignorées et qui luttent contre les multiples formes d'oppression, entre autres, la violence faite aux femmes et aux minorités, l'imposition de savoirs venus d'ailleurs, l'assujettissement à un savoir imposé ou la sujétion à un savoir hégémonique non inclusif. " lequel les femmes sont responsables des violences dont elles sont les victimes. Par ailleurs, les intervenantes en VFF montrent que dans une société démocratique comme la nôtre, il n'y a pas de place pour un tel discours et qu'il doit être remplacé par celui de la responsabilité individuelle, collective et sociale en faveur de l'éradication de toutes les formes deVFF.

Pour nous, il s'agit d'un privilège que de pouvoir montrer pour une toute première fois comment les intervenantes enVFF en Ontario français construisent leurs connaissances. Certes, cette construction reste inachevée. Par contre, elle permet de donner la parole à celles qui sont souvent ignorées et qui luttent contre les multiples formes d'oppression, entre autres, la violence faite aux femmes et aux minorités, l'imposition de savoirs venus d'ailleurs, l'assujettissement à un savoir imposé ou la sujétion à un savoir hégémonique non inclusif. Et même si l'analyse n'en est qu'à ses débuts, qu'elle est en construction et que nous n'avons pas toutes les réponses, elle se poursuit...

\section{Notes}

1 Les organismes membres d'AOcVF sont des centres d'aide et de lutte contre les agressions à caractère sexuel (CALACS), des maisons d'hébergement pour femmes violentées et des programmes en matière de violence faite aux femmes et aux enfants qui offrent des services en français à travers l'Ontario.

2 Analyse de contenu thématique de tous les comptes rendus des rencontres semestrielles des intervenantes des CALACS et des maisons d'hébergement de 2005 à 2013; analyse de contenu thématique de tous les comptes rendus des rencontres du Conseil d'administration d'AOcVF de 
2005 à 2013; deux courts sondages menés auprès des intervenantes participant aux rencontres régulières d'AOcVF; résultats de quelques évaluations de femmes qui ont utilisé des services en français entre 2010 et 2013; consultations entre les chercheuses, le comité organisateur des États généraux de 2014, les membres du conseil d'administration d'AOcVF et les organismes membres, tous invités à lire le document afin d'offrir une rétroaction et de valider son contenu; recension et une analyse de la documentation qui a été produite depuis une dizaine d'années sur les enjeux liés à la VFF et sur les services en français (Garceau et Sirois, 2014).

3 Une partie importante des documents produits par AOcVF peut être consultée sur le site http:// francofemmes.org/aocvf/index.cfm?Repertoire_No=-1051436664\&Voir=menu\&M=3975

4 Shelagh Day est la présidente fondatrice du Legal Education and Action Fund, éditrice de Canadian Human Rights Reporter et récipiendaire de la Médaille du Gouverneur Général commémorant l'affaire "personne » en 2008 et membre de l'Ordre du Canada en 2014.

5 La Coalition est formée de la Canadian Association of Sexual Assault Centres, l'Association des femmes autochtones du Canada, l'Association canadienne des Sociétés Elizabeth Fry,AOcVF, La Concertation des luttes contre l'exploitation sexuelle, le Regroupement québécois des centres d'aide et de lutte contre les agressions à caractère sexuel et le Vancouver Rape Relief.

6 Mémoire présenté par la Coalition à la Cour d'appel de l'Ontario, Affaire Bedford c. Canada, sur le site http://www.abolitionprostitution.ca/francais/qui-sommes-nous, réf. du 15 octobre 2014.

7 Le gouvernement fédéral a adopté en novembre 2014 la Loi sur la protection des collectivités et des personnes victimes d'exploitation qui criminalise les clients et proxénètes, sans toutefois criminaliser l'offre de services sexuels, à moins que celle-ci ne se fasse à la proximité de lieux où se trouvent des enfants, comme les écoles par exemple.

8 Voir le site : http://www.stopthetraffik.org/library

9 Voir le site : http://www.adultentertainmentassociation.ca/

10 Les femmes victimes de violence se présentent souvent devant les juges sans le soutien d'une avocate ou d'un avocat. Selon un rapport rédigé en 2013 par la Commission du droit de l'Ontario, " entre 50 et $70 \%$ des parties ne sont pas représentées » dans les litiges au Tribunal de la famille (Commission du droit de l'Ontario, 2013).

\section{Bibliographie}

ALLIANCE CANADIENNE FÉMINISTE POUR L'ACTION INTERNATIONALE (2008). Inégalité des femmes au Canada, Mémoire de l'Alliance canadienne féministe pour l'action internationale au Comité des Nations Unies sur l'élimination de toutes les formes de discrimination à l'égard des femmes: à l'occasion de l'examen des $6^{e}$ et $7^{e}$ rapports $d u$ Canada, Ottawa, Alliance canadienne féministe pour l'action internationale.

BISONNETTE, Sophie (2007). Sexy Inc. Nos enfants sous influence, Montréal, Office national du film. BRUCKERT, Chris, Colette PARENT et Danielle POULIOT (2006). Comment répondre aux besoins des travailleuses du sexe de rue dans la région d'Ottawa-Gatineau, Ottawa, Centre Sophie Espoir. 
BRUNET, Lucie, et collab. (1998). Les services en français en matière de VFF : le défrichage d'un chemin tortueux. Profil des services en français en matière de VFF et identification des défis et des besoins dans la prestation de services en français, Ottawa, Action ontarienne contre la VFF.

BRUNET, Lucie, David SHERWOOD et Marie-Luce GARCEAU (2009). Étude de besoins sur les services en français pour les victimes francophones de Kingston, Toronto, ministère du Procureur général de l'Ontario, [document non publié].

BRUNET, Lucie, et Marie-Luce GARCEAU (2004a). Faire autant avec si peu... Bilan et profil des services en français en matière de violence contre les femmes (1994-2004), Ottawa, Action ontarienne contre la VFF.

BRUNET, Lucie, et Marie-Luce GARCEAU (2004b). États généraux 2004. Développement des services en français en matière de violence contre les femmes. Rapport des États généraux, Ottawa, Action ontarienne contre laVFF.

BRYDON-Miller, Mary (2001). "Education, research and action: Theory and methods of participatory aAction research ", dans Deborah L. Tolman et Mary Brydon-Miller (dires.), From subjects to subjectivities: A handbook of interpretative and particpatory models, New York, New York University Press, p. 76-89.

CANADIAN CENTRE FOR POLICY ALTERNATIVES (2012). A budget for the rest of us, alternative federal budget 2012, Ottawa, Canadian Centre for Policy Alternatives, Ottawa.

COALITION DES FEMMES POUR L'ABOLITION DE LA PROSTITUTION (2013). Mémoire présenté à la Cour suprême du Canada par la Coalition des femmes pour l'abolition de la prostitution, réf. du 15 septembre 2014, http://www.abolitionprostitution.ca/francais/documents-legaux

COLLOQUE PROVINCIAL SUR LES AGRESSIONS À CARACTÈRE SEXUEL, COLLECTIF (1994). Sensibiliser, décider, agir. Les Actes du colloque provincial sur les agressions à caractère sexuel, Ottawa, Colloque provincial sur les agressions à caractère sexuel.

COMITÉ PERMANENT DE LA JUSTICE ET DES DROITS DE LA PERSONNE (2006). Le défi du changement : Étude des lois pénales en matière de prostitution au Canada, réf. du 10 septembre 2014, http://www.parl.gc.ca/Content/HOC/committee/391/just/reports/rp2599932/justrp06/ sslrrp06-f.pdf

COMMISSION DU DROIT DE L'ONTARIO (2013). L'amélioration de l'accès à la justice familiale à des points d'entrée globaux et à l'inclusivité : Rapport final - Février 2013, Partie I, Section II : Aperçu du système de justice familiale, réf. du 13 septembre 2014, http://lco-cdo.org/fr/familylaw-reform-final-reporthttp://lco-cdo.org/fr/family-law-reform-final-report

CORBEIL, Christine, et Isabelle MARCHAND (2010). "L'intervention féministe : un modèle et des pratiques au cœur du mouvement des femmes québécois ", dans Christine Corbeil et Isabelle Marchand (dires.), L'intervention féministe d'hier à aujourd'hui. Portait d'une pratique diversifiée, Montréal, les éditions du remue-ménage, p. 23-56.

DAY, Shelagh (2008). La prostitution, une violation des droits humains des femmes pauvres, Ottawa, Action ontarienne contre la VFF, 9 p.

DE BEAUVOIR, Simone (1949). Le deuxième sexe, Paris, Éditons Gallimard.

DUFOUR, Rose (2005). Je vous salue... Le point zéro de la prostitution, Montréal, Éditions MultiMondes, $672 \mathrm{p}$.

ÉQUIPE PRAXIT (2011). «Co-construire les connaissances », Revue du Cremis,Vol. 4, № 2, p. 38-43. 
GARCEAU, Marie-Luce, et Marc CHARRON (2001). Pour exprimer son histoire en français. Rechercheaction sur les besoins, les services et les modèles de prestation de services en français en matière de violence contre les femmes dans le nord de l'Ontario, Thunder Bay, Centre des femmes francophones du Nord-Ouest de l'Ontario.

GARCEAU, Marie-Luce, Sébastien SAVARD et Stéphane RICHARD (2012). "Une lutte constante pour contrer les violences faites aux femmes et leur assurer des services en français de qualité. Entrevue avec Ghislaine Sirois ", Reflets, revue d'intervention sociale et communautaire, Vol. 18, $\mathrm{N}^{\circ}$ 1, p. 22-37.

GARCEAU, Marie-Luce, et Ghislaine SIROIS (2014). Éliminer la VFF : une tâche ardue. États généraux 2014. État des lieux sur les agressions à caractère sexuel et la violence conjugale : services en français, enjeux prioritaires dix ans après les États généraux de 2004, Ottawa, Action ontarienne contre la VFF.

HAUT-COMMISSARIAT DES NATIONS UNIES (1993). Déclaration sur l'élimination de la violence à l'égard des femmes, réf. du 30 octobre 2013, http://www2.ohchr.org/french/law/femmes_violence.htm

LAMONT, Ève (2010). L'imposture [vidéo], Montréal, Les Productions du Rapide Blanc.

LiAMPUTTONG, Pranee (2007). Researching the Vunerable - A Guide to Sensitive Research Methods, London, Sage Publication, $256 \mathrm{p}$.

MCINTURFF, Kate (2013). The gap in the gender gap, Ottawa, Centre canadien de politiques alternatives, $46 \mathrm{p}$.

PRESLEY, Laureen (2005). Whose knowledge is it anyway? Feminist epistemology and science - An annotated bibliography, [Document submitted to Dr. Jim V. Carmichael], Department of Library and Information Studies, School of Education, University of North Carolina.

PUIG DE LA BELLACASA, Maria (2003). "Scientificité et politique d'aujourd'hui : un regard féminise ", Nouvelles questions féministes, Vol. 22, No 1, p. 48-60

ROBERT, Jocelyne (2005). Le sexe en mal d'amour, de la révolution sexuelle à la régression érotique, Montréal, les Éditions de l'Homme, 240 p.

RISSE, David, et Katja SMEDSLUND (2014). «Conclusion », dans Katja Smedsluind et David Risse (dirs.), Responsabilités et violences envers les femmes, Québec, Presses de l'Université du Québec, p. 331-341.

ROMITO, Patrizia (1997). "Violences contre les femmes : des réponses féministes ", Nouvelles Questions Féministes, Vol. 18, № 2, p. 35-58.

VERMEIREN, Marie (2006). Not for sale/Pas à vendre [documentaire vidéo], Lobby Européen des Femmes (LEF) et de la Coalition contre le trafic des Femmes (CATW).

WARIDEL, Laure (2012). Co-constuire un savoir qui change le monde, Montréal,Association canadienne pour le savoir, Montréal, $80^{\mathrm{e}}$ congrès de l'ACFAS. 